\section{Dr. Tselios, et al, reply}

\section{To the Editor:}

We thank De Luca and colleagues ${ }^{1}$ for their interest in our paper ${ }^{2}$. We agree with the authors that the pathophysiologic mechanisms for abnormal cardiac biomarkers in systemic lupus erythematosus (SLE) are many and may include both inflammatory and ischemic causes among others. We also agree that these conditions may present subclinically. In the paper, we reported that even in the patients who presented with no symptoms at all, further investigations (instigated by the incidental finding of abnormal cardiac biomarkers) revealed myocarditis $(n=1)$, coronary artery disease $(n=1)$, uncontrolled systemic hypertension $(n=1)$, or even pulmonary hypertension $(\mathrm{n}=2)$.

Regarding the 6 patients with antimalarial-induced cardiomyopathy (AMIC), the diagnosis was confirmed by endomyocardial biopsy (EMB) in three. The other 3 were characterized as "possible" based on structural (septal and left ventricular hypertrophy) and functional (diastolic dysfunction) abnormalities compatible with AMIC. Moreover, other causes were excluded by echocardiography, coronary angiography, and cardiac magnetic resonance (CMR) imaging. They had no concomitant clinical or serological activity that might have indicated active SLE, and antimalarial withdrawal led over time to a significant decrease or normalization of the cardiac biomarkers along with regression of the left ventricular hypertrophy.

Concerning the other 5 patients for whom a definitive diagnosis could not be made, they are closely monitored and no changes (clinical, biochemical, or echocardiographical) have been observed after 18 months of followup. Regarding the potential implication of antiphospholipid-related thrombotic microangiopathy, none of these 16 patients was positive for anticardiolipin antibodies or lupus anticoagulant at the time of the study.

Should EMB be performed in these patients and should it be mandatory in all patients with systemic autoimmune diseases and elevated cardiac biomarkers? The answer is probably not straightforward. Traditional (echocardiography, coronary angiography) and modern diagnosing techniques (CMR with T1/T2 mapping, positron emission tomography) would suffice in the majority of patients. In addition, the issues of feasibility and sampling error of the EMB should be considered in certain settings. For example, in our recent report on lupus myocarditis (reporting on 147 patients with clinically overt disease), normal pathology findings were reported in $5 / 8$ patients with severely compromised left ventricular systolic function ${ }^{3}$. This implies that myocardial damage in this condition is patchy and not diffuse, thus limiting the value of EMB. Despite the well-established prognostic significance of these biomarkers ${ }^{4}$, EMB provided a diagnosis in $25.5 \%$ and changed clinical course in $22.7 \%$ of 851 patients with unexplained heart failure ${ }^{5}$. Further, Yoshida, et al showed that a combination of clinical data, echocardiography, and CMR achieved better sensitivity and specificity than EMB in 136 patients with hypertrophic cardiomyopathy ${ }^{6}$.

Nevertheless, heart biopsy is a powerful tool in patients for whom the diagnosis remains elusive, and it represents the gold standard for the precise diagnosis of AMIC in particular. However, in that context, we believe that antimalarial withdrawal (the only available intervention apart from supportive treatment) should not be delayed if there is a strong clinical suspicion.

We agree with De Luca and colleagues that these findings ${ }^{1,7}$ deserve further research to elucidate the relevant pathophysiologic mechanisms and their clinical significance in patients with systemic autoimmune diseases. In the interim, screening with cardiac biomarkers for subclinical AMIC should be carried out in patients using antimalarials for 5 or more years.

KONSTANTINOS TSELIOS ${ }^{\circ}$, MD, PhD, Toronto Lupus Clinic, Centre for Prognosis Studies in Rheumatic Diseases, University Health Network; DAFNA D. GLADMAN ${ }^{\circ}$, MD, FRCPC, Toronto Lupus Clinic, Centre for Prognosis Studies in Rheumatic Diseases, University Health Network; PAULA HARVEY, BMBS, PhD, FRACP, Cardiology Department, Women's College Hospital, University of Toronto; SHADI AKHTARI, MD, FRCPC, Cardiology Department, Women's College Hospital, University of Toronto; JIANDONG SU, MB, BSc, Toronto Lupus Clinic, Centre for Prognosis Studies in Rheumatic Diseases, University Health Network; MURRAY B. UROWITZ ${ }^{\circ}$, MD, FRCPC, Toronto Lupus Clinic, Centre for Prognosis Studies in Rheumatic Diseases, University Health Network, Toronto, Ontario, Canada. Address correspondence to Dr. M.B. Urowitz, University of Toronto Lupus Clinic, Centre for Prognosis Studies in the Rheumatic Diseases, Toronto Western Hospital, 399 Bathurst St., 1E-410B, Toronto, Ontario M5T 2S8, Canada.

E-mail:m.urowitz@utoronto.ca

\section{REFERENCES}

1. De Luca G, Campochiaro C, Dagna L. Abnormal cardiac biomarkers in patients with systemic lupus erythematosus and no prior heart disease: the role of endomyocardial biopsy. J Rheumatol 2019;46:1421-2.

2. Tselios K, Gladman DD, Harvey P, Akhtari S, Su J, Urowitz MB. Abnormal cardiac biomarkers in patients with systemic lupus erythematosus and no prior heart disease: a consequence of antimalarials? J Rheumatol 2019;46:64-9.

3. Tanwani J, Tselios K, Gladman DD, Su J, Urowitz MB. Lupus myocarditis: a single center experience and a comparative analysis of observational cohort studies. Lupus 2018;27:1296-302.

4. York MK, Gupta DK, Reynolds CF, Farber-Eger E, Wells QS, Bachmann KN, et al. B-type natriuretic peptide levels and mortality in patients with and without heart failure. J Am Coll Cardiol 2018;71:2079-88.

5. Bennett MK, Gilotra NA, Harrington C, Rao S, Dunn JM, Freitag $\mathrm{TB}$, et al. Evaluation of the role of endomyocardial biopsy in 851 patients with unexplained heart failure from 2000-2009. Circ Heart Fail 2013;6:676-84

6. Yoshida A, Ishibashi-Ueda H, Yamada N, Kanzaki H, Hasegawa T, Takahama H, et al. Direct comparison of the diagnostic capability of cardiac magnetic resonance and endomyocardial biopsy in patients with heart failure. Eur H Heart Fail 2013;15:166-75.

7. Bosello S, De Luca G, Berardi G, Canestrari G, de Waure C, Gabrielli FA, et al. Cardiac troponin T and NT-proBNP as diagnostic and prognostic biomarkers of primary cardiac involvement and disease severity in systemic sclerosis: a prospective study. Eur J Intern Med 2019;60:46-53.

First Release July 1 2019; J Rheumatol 2019;46:10; doi: $10.3899 /$ jrheum.190255 